\title{
COMMISSION 42: CLOSE BINARY STARS
}

\section{(ETOILES DOUBLES SERREES)}

\author{
PRESIDENT: Edward F. Guinan \\ VICE-PRESIDENT: P. Szkody \\ ORGANIZING COMMITTEE: M. Rodonó (Past President), L. Bianchi, \\ J.V. Clausen, H. Drechsel, G. Hill, S.J. Kenyon, V.S. Niemela, Y. Osaki, \\ C.L. Sterken (IBVS), R.E. Taam, O. Vilhu, J.C. Wheeler \& J.H. Wood
}

\section{INTRODUCTION: E.F. Guinan}

\subsection{Close Binaries at the Dawn of the New Millennium}

This is the last triennial report of Commission 42 for this millennium. A great deal has been accomplished in the study of Close Binary Stars (CBS) since the discovery of the first close (eclipsing) binary, Algol, in 1783 by John Goodricke. Now, over 10,000 CBS (most eclipsing variables) are known. More than 5000 of these CBS were discovered over the last several years alone! And many more are expected to be detected over the next few years. Most of these stars were found as spin-offs of microlensing surveys. Interestingly, nearly half of these stars are found outside our Galaxy, primarily in the Magellanic Clouds and M31. Every type of star is represented as a member of a close binary. These include main sequence (as well as pre-main sequence) stars, giants, and supergiants, with the entire possible range of of spectral types and masses represented. Moreover, "dying" stars and "dead" stars, such as white dwarfs, neutron stars, black holes, and, more recently, even brown dwarfs and giant planets (e.g., $51 \mathrm{Peg}$ ) have been found as members of close binary systems.

As we start the new millennium, the future looks very bright for the study of close binary stars (CBS). The contributions in this triennial report emphatically attest to the vitality and wide range of interesting and important research being done on the many different kinds of CBS. Close binaries continue to play vital roles in many areas of modern astronomy and astrophysics. In particular, eclipsing binaries continue to provide essential fundamental data, on masses, radii, and luminosities of stars. These data are fundamental to understanding all stars, as well as the clusters and galaxies in which they reside, and the basic physical laws that govern their behavior. As important as stellar masses and radii are to astrophysics, CBS are important and interesting for many other reasons.

CBS can serve as astrophysical laboratories for investigating a wide range of physical phenomena that include the internal structure and convective-core overshooting in stars. Also, specially suited CBS are important for studying stellar atmospheres from limb darkening and gravity darkening determinations, as well as from atmospheric eclipses and eclipse mapping techniques. Some close binaries with cool components (e.g., RS CVn, W UMa and some Algol-type systems) have proven very useful in studying dynamo-related magnetic activity, that include starspots and chromospheric and coronal structures.

Evolved close binaries, in particular binary pulsars, are providing important tests of general relativity. For example, long term observations of the binary pulsar PSR 1913+16 are consistent with the predictions of general relativity. Also, CBS having neutron star or black hole components are providing crucial insights into the physical properties and physics of these rare objects that can be obtained in no other way. 
Semi-detached binary systems (such as Algols and CVs) with accretion disks can serve as well-constrained analogs for understanding accretion processes in Young Stellar Objects (YSOs) (and their proto-planetary disks), and also in understanding accretion processes associated with massive black holes now believed to be located in galactic nuclei, and responsible for powering AGN-galaxies and quasars.

As once mentioned by Virginia Trimble at a binary star symposium, some stars are born great, while others achieve greatness, or have it thrust upon them. The later case is true for some "ordinary" stars that happen to be members of a close binary. Most stars, if single, whould end their lives as white dwarfs and fade inconspicuously away like "old soldiers". But, if they are members of close binaries, these stars could exit in a "blaze of glory", becoming supernovae (SNe). This is the case for Type-I supernovae (SNIa) in which the white dwarf component of the CBS accretes sufficient matter from its nearby companion to reach the Chandrasekhar limit $\left(\mathrm{M}=1.4 \mathrm{M}_{\odot}\right)$ and undergo a spectacular, catastrophic detontation. Over the last decade, SNIa's have become extremely important to cosmology, serving as standard candles for gauging the distances to distant galaxies and for determining the nature of our universe. It is possible that some symbiotic binaries may result in supernovae, if the white dwarf member can gain enough mass from the wind of its M-giant companion to reach the Chandrasehkar limit.

Although less spectacular than supernovae, nova and other cataclysmic binary stars owe their large light variations to accretion onto their white dwarf members from low mass, cool companions. These systems are important proving grounds for studying stellar evolution, accretion processes, and plasma physics. Finally CBS, consisting of close pairs of neutron stars (or black hole-neutron star close pairs) have been proposed to explain the huge energy outbursts observed in gamma-ray bursters (e.g., GRB970508) as the two neutron stars merge.

\subsection{Conference Proceedings and Books Related to Close Binary Systems}

As demonstrated by the number and quality of the papers appearing in the literature on close binary stars (CBS) over the last three years (several hundred papers/year - see Bibliography on Close Binaries), the field is very vigorous and still growing. Important reviews on various aspects of CBS research are included in the following publications: The Third Pacific Rim Conference on Recent Developments on Binary Star Research (ASP Conf. Ser. 130), Visual Double Stars: Formation and Evolution Tracks (Kluwer, 1997), Wild Stars in the Old West: Proceedings of the 13th North American Workshop on Cataclysmic Variables and Related Objects (ASP Conf. Ser. 137), Pacific Rim Conference on Stellar Astrophysics (ASP Conf. Ser. 138), Cool Stars, Stellar Systems, and the Sun, Tenth Cambridge Workshop (ASP Conf. Ser. 154), Annapolis Workshop on Magnetic Cataclysmic Variables (ASP Conf. Ser. 157), High Energy Processes in Accreting Black Holes (ASP Conf. Ser. 161), Theory and Tests of Convection in Stellar Structure (ASP Conf. Ser. 173), Eclipsing Binary Stars: Modelling and Analysis (Springer-Verlag, 1999), Stellar Evolution in Real Time (New Astro. Reviews 19), and Variable and Binary Stars as Astrophysical Tests (NATOASI Series; Kluwer, in press). Additional related publications on CBS are given in the Division V report.

\subsection{Rationale, Organization and Acknowledgments:}

The present $\mathrm{C} 42$ report is different from previous ones in that it is more concise, and, because of this, the report cannot, and does not, attempt to address every aspect of current research in close binaries. To discuss all the work on close binaries would require much more space than allowed in this volume and be a burden on the contributors. Moreover, it is not really necessary to have comprehensive reports these days because information and papers on CBS can be obtained over the internet and World Wide Web from various sites (e.g., ADS/SIMBAD). Furthermore, a comprehensive bibliography (with notes) of papers and research that relate to close binaries is given in the Bibliography of Close Binaries (see 
Sec. 2). We owe a great debt to the Editor-in-Chief of the BCB, Dr. Horst Dreschel, and the members of the editorial board for performing such an important service for the binary star community. Also, many papers on CBS appear in a timely fashion in the Information Bulletin of Variable Stars (IBVS). The IBVS now can be accessed at the Konkoly Observatory Web site. Again, C42 is very grateful to Chris Sterken and the Chiefeditors of this important resource.

In this report the invited contributors were encouraged to express their opinions and ideas on what they considered the major advances in their speciality during the last three years. The authors were also encouraged to speculate about major developments they expect in CBS research for the future and to comment on some of the major unsolved problems relating to CBS. Also, the contributors were requested to comment on the impact new technologies, instrumentation, telescopes (both on the ground and in space) could have on close binaries research.

As President, I want to thank the C42 OC members for their assistance and advice during the last 3 years. I also wish to thank the invited contributors of the Report for taking the time and making the effort to write their valuable and insightful discussions and comments.

\section{BIBLIOGRAPHY OF CLOSE BINARIES: H. Drechsel}

C42 continued its bibliographical service by regular, semi-annual publication of its Bibliography of Close Binaries (BCB). Effective 1998 (issue 66 on), the former title Bibliography and Program Notes on Close Binaries $(B P N)$ has been changed into BCB. The bibliography is thought to assist CBS researchers in their efforts with literature search, reviews of the field, and alerts about new developments.

The editorial board currently consists of D.R. Faulkner, T.J. Herczeg, V.G. Karetnikov, E. Lapasset, C. Maceroni, Y. Nakamura, R.G. Samec, C.D. Scarfe, M. Vetesnik, M. Wolf, and editor-in-chief $\mathrm{H}$. Drechsel.

This bibliography is a collection of references for CBS-related publications in all major journals. Each issue typically contains a total of 500-600 entries. Different sections list publications on individual stars, topics of general interest for CBS research (mostly of theoretical nature), and collections of data like, e.g., new catalogues, surveys, or statistical investigations; proceedings of conferences, symposia, and monographs are also covered. The individual bibliographic entries are categorized according to their principal nature, i.e., presentation of observational data of all kinds, discussion of derived physical data, introduction of new observational techniques, or theoretical or statistical investigations.

BCB issues are available from: http://www.sternwarte.uni-erlangen.de/ftp/bcb or can be downloaded via anonymous ftp from: ftp://www.sternwarte.uni-erlangen.de/pub/bcb. Hardcopy versions are also distributed free of charge upon request (drechsel@sternwarte.unierlangen.de).

\section{METHODS \& TECHNIQUES}

\subsection{Progress on Modeling Light Curves of Eclipsing Binaries: R. E. Wilson}

A program that computes light curves can be generalized to compute numerous other observable quantities with modest extra programming. It also can compute light curve phenomena that go beyond ordinary physical situations. Some generalizations make little drain on computer resources, while others do require considerable speed and memory, but well within the capabilities of current machines.

- Advances in Modeling: Light curves of fluorescent binaries: The main current application is to symbiotic stars, especially symbiotic novae, but any binary with a fluorescent wind, chromosphere, or coherent circumstellar flow is a potential application. Representation of individual lines may be unnecessary, as the number of lines is 
typically large and their combined brightness can be modeled statistically. Coherent scattering can be included with somewhat similar algorithms. Circumstellar attenuation: Certain highly evolved binaries with large scale mass flow contain circumstellar regions that attenuate photospheric light. An example is AX Monocerotis, where the photometric effects in the ultraviolet are rather spectacular. Such light curves can now be computed for essentially arbitrary matter distributions, including regions with density structure, by inclusion of semi-transparent clouds in the model. Some guidance on modeling light curves is given in the recent book Eclipsing Binary Stars: Modeling and Analysis by Kallrath and Milone.

- Polarization: Polarization curves for combined effects of circumstellar scattering and photospheric electron scattering can now be computed. Progress has halted for lack of suitable observations. Part of the problem is that observational polarimetric papers quite often contain no data tables. It is not obvious why non-publication of data is particularly a problem in this sub-field. Another practical problem - perhaps unavoidable - is that the quantity of observations is typically 10 to 100 times smaller than for photometry. Designers of polarimeters can help by making speed of operation a priority. To a lesser extent, small data quantities are also a problem in most radial velocity work.

- Spectral line profiles and rotation: Programs that generate absorption and emission line profiles, including all the ordinary binary star effects, are now available. It is no longer necessary to assume simple geometrical models when extracting rotation from profiles. Stellar atmosphere simulation capabilities for a wide range of temperature and $\log g$ have been developed by several groups. This advance is very important for proper modeling of highly evolved stars, especially where the two star temperatures are considerably different.

- Analyzing observations: Ephemeris quantities (reference epoch, period, $\mathrm{dP} / \mathrm{dt}$, etc.) have traditionally been dealt with in a preliminary analytic stage and the results used to compute phases for a second stage. A conceptually simple but productive improvement is to enter observation times rather than phases as direct input to the overall problem and solve for all parameters together. Simultaneous solutions of several kinds are on the way or already developed, including light, radial velocity, and visual (equivalently speckle or interferometric) observations. Additional kinds could be developed if enough data sets for suitably conditioned binaries existed. There certainly will be more such data because of new interferometric facilities.

- Overview points and recommendations: In several developing areas there are disjoint communities of astronomers working on closely related problems who are not well acquainted with each others work. For example, workers on symbiotic stars divide into mainly spectroscopic and mainly photometric camps. Typical work emphasizing spectroscopy treats eclipses and related photometric phenomena at the level of free-drawn circles, while typical work that emphasizes photometry may not properly incorporate spectra at all, or only in a cursory way. A similar situation obtains for $\mathrm{X}$-ray binaries. IAU commissions could take the lead in bringing such communities together.

\subsection{Techniques for Analyzing CBS Observations: P. Harmanec}

In addition to the cross-correlation technique developed earlier, there has also been progress in the development and practical application of other novel methods of analyses of spectra of spectroscopic binaries:

- Tomographic separation of composite spectra (ApJ 423, 446) assumes a priori knowledge of the RV curves and allows reconstruction of individual line profiles, therefore an objective spectral classification of both binary components. It has recently been 
applied to several complicated hot binaries (ApJ 483, 439; ApJ 485,350;ApJ 506,424; ApJ 518,450).

- Doppler tomography also assumes the knowledge of RV curves and allows the mapping of the distribution of circumstellar matter within the interacting binary systems. It was mainly applied to Algol binaries (ApJ 493, 326; ApJS 123, 537).

- Especially notable is the disentangling technique KOREL, developed by Hadrava (A\&AS 122, 581; A\&AS 122, 581). The only assumption inherent to the current version of this method is that the line profiles have constant shape (their intensity can vary). The technique uses the observed electronic spectra and returns the orbital elements (including apsidal motion and orbital period change) and also individual line profiles for a hierarchical system of up to 5 stars. This way, it also allows an objective removal of telluric lines from the red and IR spectra (solving for the Earth motion around the Sun as one of the components of the system). Its practical application confirmed that even with a spectrograph of a moderate resolution of 15000 , the lines of a secondary which is 4 mag fainter than the primary can be detected and measured (A\&A 345, 855).

Harmanec (A\&A 335, 173) published formulae for a reliable transformation of Hippar$\cos H_{p}$ magnitudes into Johnson $V$ and $B$ magnitudes which allows an easy combination of existing $U B V$ photometry of binaries (and other variables) with the precious Hipparcos data, free of 1-d aliases.

The Ondrejov group, collaborating with colleagues from other places, launched the SEFONO program (SEarch for FOrced Non-radial Oscillations) in an effort to find evidence of rapid line-profile changes in the spectra of hot, rapidly rotating stars in binary systems with eccentric orbits. So far, such variations were detected in 3 cases (A\&A 319, 867; A\&A $322,565 ;$ A\&A 345,855$)$. A very similar program has also been started by the group at Leuven: a study of properties of $\beta$ Cep stars in binaries, again with very promising results (MNRAS 302, 245; A\&A 329,137; A\&A 324, 1096). Notable in this context is the final proof that the magnetic Be star V1046 Ori with line-profile changes is a component of an eccentric binary (A\&A 337, 183). Cross-correlation technique and disk modeling was used to derive (still rare) reliable masses for a binary with a Be component V360 Lac (A\&A 324, 736).

The presence of jet-like structures was reported for $\beta$ Lyrae on basis of spectropolarimetry (AJ 115,1576). Modelling of the light curve and continuum energy distribution showed that a scattering halo will have to be added to the model of the binary with an accretion disk to describe the observations (ApJ 509, 379). A search for small eccentricity in $\beta$ Lyrae was carried out by Wilson and Van Hamme (MNRAS 303, 736).

\subsection{Polarimetry of Close Binary Systems: R. H. Koch}

Close binary polarimetry is currently driven by a few new technologies: rather easy access to large, modern telescopes in good climates; clever and efficient designs for spectropolarimeters working at low resolution; and Earth-orbiting space platforms. As a result, wideband, visible work on main sequence stars has almost vanished because signals are low and modulations modest at best. There remains some interest in hot, evolved binaries as is indicated by the 7 which have been studied recently. Symbiotic pairs are almost as popular, with 6 systems having been analyzed over the last triennium. The real attraction, however, is ordinary and magnetic white dwarfs (and, to a lesser degree, neutron stars) with results for more than two dozen pairs appearing over the same interval. These trends should continue over the next reporting interval. Interested readers should also acquaint themselves with the section of The Commmission 25 Report wherein results for single stars are summarized. The last statement is a speculation but almost certainly well-founded. 


\section{PHYSICAL PROPERITIES OF CLOSE BINARY SYSTEMS}

\subsection{Physical Properties and Internal Structure of Stars in Eclipsing Binary Systems: A. Giménez}

During the trienium 1997-1999, the study of absolute dimensions using well-detached doublelined eclipsing binaries has continued its progress. The attention has been centered in the study of low mass stars because of a) the need to cover this region of the HR diagram with solar-like or less massive stars, and b) the extensive use Coravel-type photometric spectrometers for the determination of radial velocities using templates, only valid for late-type stars. A long standing problem of flux deficiency for the secondary stars in low mass binaries is still present despite the use of better theoretical models and more accurate observational data.

On the other hand, the analysis of moderate and massive stars has confirmed the metal deficiency of B-type stars when compared to the Sun, and the more massive cases are opening the possibility to determine temperature calibrations in a difficult region where NLTE model atmospheres are generally needed. Moreover, the analysis of evolved systems is being used for the estimation of the level of convective core overshooting, or for that matter, increased core size and mixing, needed in real stars.

Special attention has been devoted to the determination of accurate absolute dimensions in hot binaries which are members of the Magellanic Clouds. These should be the basis for the comparison of observational data with models for stars born in a different evolutionary and chemical environment than those in our Galaxy. In addition, the obtained data provides an evaluation of the distance scale which is essentially free of calibration problems.

Concerning the study of binaries in clusters, long-term surveys of radial velocities give a much better overview of their statistical distribution while some special cases of eclipsing binaries have been identified as cluster members.

The search for new eclipsing binaries has also been very much revitalized (after few new surveys since the times of photographic plates) by CCD based wide-field cameras. An especially important contribution to this problem of identifying new eclipsing binaries was provided by the photometric measurements of the Hipparcos satellite which, in addition to accurate distances leading to better calibrations, provided an unbiased photometric monitoring of many stars down to 11th mag and the discovery of many new eclipsing binaries. In fact, 917 stars were identified as eclipsing systems with well determined orbital periods, out of which 343 were totally new, despite the bright magnitudes covered by the satellite.

Research in the fields of apsidal motion tests or tidal evolution of detached binaries has not received so much attention as in previous periods but this may reflect the advances in collecting new and better data in a difficult and time consuming effort. New systems are in fact being added to the lists of available binaries with accurate determinations both for the study of internal structure and the relativistic effect. Rotational velocities are measured with increased accuracies, adding the possibility to compare evolutionary stories of the systems with tidal evolution predictions.

\section{CLOSE BINARIES WITH COOL COMPONENTS}

\subsection{The Status of Algol-type Binaries: M. Richards}

Over the past few years, several pioneering articles have been published on the topic of Algol-type binaries. These binaries have been almost forgotten as members of the group of close, interacting binaries because they are in the slow phase of mass transfer; and because of the perception that we already know all there is to know about these systems. While it is true that the prototype of the class, $\beta$ Per or Algol, has been studied for over two hundred years (and observed for thousands of years), the same is not true of other members of the class. Only a few hundred of these systems are known, and most of these are in 
the northern hemisphere. Quite often, the spectral type of the faint, late-type, secondary star is not well-determined, and distances to these objects are uncertain. These are not characteristic of a class of objects that has been studied thoroughly. Yet we must ask the question: "Why study Algols anyway?"

The answer is simple. The Algol-type binaries provide us with an opportunity to study the influence of magnetic fields on the mass transfer process. Moreover, Doppler tomograms of some short-period Algols have provided distinct images of the gas stream that follow the predicted gravitational path. No other class of binaries has produced such information. Since these binaries are in the slow phase of mass transfer, we can study the gas stream itself, as well as the active process of mass transfer, instead of only the final products of mass transfer (e.g., the accretion disk). The Algols can provide the critical estimates of mass transfer rates that are needed to produce realistic theoretical models. In the wider Algols, the gas stream from the magnetically-active mass loser has enough room to form a classical accretion disk but, in the short-period systems, the gas stream makes direct impact with the mass-gaining star. At least two short-period Algols have been found in which the accretion regions are quite active; changing within an orbital cycle from a gas stream distribution to one resembling an accretion disk.

Within the past few years, the study of the Algol-type binaries has been revitalized in several ways: the systematic collection of multiwavelength (mostly $\mathrm{H} \alpha$ ) spectra at positions around the entire orbit of up to 20 binaries; the thorough analysis of these observed spectra and difference spectra obtained with the aid of model atmospheres calculations; the use of Doppler tomography to generate reconstructed images of emission sources in the binary; hydrodynamic simulations of the mass transfer process; a long-term continuous radio flare survey to determine the frequency of flaring activity; and the completion of a 23-year spectroscopic study of the non-eclipsing Algol called CX Dra. In the next decade, there is still a lot of work to be done. We need to study the group of non-eclipsing Algols (only six have been confirmed); determine whether the presence of a mass-losing, magnetically-active companion has any influence on the process of mass transfer; collect additional complete infrared light curves of these systems to provide information about starspot activity; obtain infrared spectra of these systems to determine the structure of circumbinary gas; obtain continuous coverage of these binaries around the orbit by establishing a round-the-Earth spectroscopic campaign using similar telescopes and detectors; and also to finally resolve these binaries using the Keck interferometer or the proposed Space Interferometer. It is clear that, even after two centuries, we have only just begun to understand the Algols and the class of interacting binaries as a whole.

\subsection{Developments in Chromospherically Active Binaries: O. Vilhu}

Close binaries continue being excellent probes of chromospheric-coronal (magnetic) activity in late type stars at high rotational rates. This is due to tidal synchronism in close binaries and rapid spin-down of single stars after their birth from magnetic braking, a consequence of this activity. Magnetic braking is also one of the key factors regulating close binary evolution, leading to angular momentum loss and reduction of the orbit. This is discussed recently by Maceroni (Turk. J. Phys., 23, 289). Present $U V$ and X-ray satellites with their good spectral resolution or broad-band energy coverage have considerably improved our knowledge of the chromospheric-coronal structures and heating mechanisms.

Using the Goddard High Resolution Spectrograph (GHRS) of the Hubble Space Telescope (HST), bimodal line profiles of the CIV 1550 line, formed at $10^{5} \mathrm{~K}$, were discovered in active binary and single stars by Wood, Linsky \& Ayres (ApJ 478, 745) and by Vilhu et al. (AJ, 115, 1610). The broad component (correlating with rotation) was interpreted as arising from microflaring, a possible heating agent. The EUVE high resolution Atlas (ApJS 113, 131) and the differential emission measure DEM(T)-curves determined for e.g., Capella and $\xi$ UMa by Mewe, Kaastra, Schrijver and collaborators are essential tools to understand coronal structures in solar type, slow and rapid rotators, both single and binary stars (see several Utrecht preprints starting 1996). The high temperature compo- 
nent seen in many cases needs to be confirmed by higher energy spectroscopy missions like ASTRO-E. However, hard, non-thermal tails seen during strong flares may be common as observed by Beppo-SAX in Algol and UX Ari by Favata \& Schmitt, (ESTEC-Preprint: ESLAB 1999/006/SA; and by Pallavicini \& Tagliaferri, in The Active X-ray Sky, [Elsevier, in press]).

Additional recent information on magnetic activity in close binary stars can be found in the review by Klaus Strassmier on Magnetic Activity in Binary Stars presented at the 12th EARN School in Tenerife during September 1999. The paper is available on the University of Vienna Web site.

\subsection{Contact Binaries at the Start of the New Millennium: C. Maceroni}

In the field of contact binaries, the last years have primarily been dominated by the release of new important databases and by their analyses. The by-products of microlensing searches yielded new homogeneous catalogs, as the OGLE-II Variable Catalog for the SMC (AcA 48,563 ), which followed the OGLE-I Catalog for the Baade's Window, and that by the MACHO experiment for the LMC (AJ 114,326). These are complementary to each other, as the Magellanic Clouds catalogs are expected to contain mainly hot contact binaries, at variance with the Baade's Window surveys.

Moreover, there were a number of discoveries of new contact binaries in clusters (see the OGLE experiment homepage for a listing: http://sirius.astrouw.edu.pl). The comparison of contact binaries in old open clusters and the Baade's window by Rucinski (AJ 116,2998) showed that late-type contact binaries have essentially the same luminosity function of the solar neighborhood-MS stars and that their relative frequency is much higher than previously accepted, an occurrence that - if confirmed - might be related to the properties of their angular-momentum-loss driven evolution. In the next few years we expect to get a better information about the chromospheres and coronae of these stars from space experiments such as EUVE and Chandra, as in the interesting case of a partially resolved coronal structure for 441 Boo by Brickhouse \& Dupree (ApJ 502,918).

It has to be said that many more efforts have recently been devoted to observation and data analysis than to theoretical studies on the internal structure and evolution of contact binaries. This is a field that suffered from stagnation in the past decade and needs further development in the future. The availability of more and more powerful computers gives hope that the intrinsic difficulties of the modeling - mainly related to the geometry of the fast rotation of such stars - will be overcome in the not too distant future.

\subsection{Research on contact binaries 1996-1999: H. Duerbeck}

Photometric observations of newly discovered and well-established contact systems, and analyses along the lines of the Wilson-Devinney method have become customary, and have been carried out by researchers in many countries. Especially valuable have been analyses of poor-contact systems (MNRAS 291,749, MNRAS 296,100). An attempt to determine the gravity darkening in a large sample of contact systems yielded values which are close to those predicted by theory (A\&A 335,199). Radial velocity studies of 20 systems, based on spectral broadening functions, have been published or are in preparation (AJ 118,515). Hipparcos parallaxes have been used to re-calibrate the luminosity-period-color relation (PASP 109,1340); Hipparcos photometry has also revealed a number of bright contact binary candidates with low inclination angles (IBVS 4513).

The variable star data bases of various dark matter surveys (OGLE, MACHO) have been especially fruitful; these large samples show a continuation of the W UMa sequence to periods 1.3-1.5 days in Baade's window (AJ 115, 1135); and blue high-mass contact binaries with orbital periods up to 2-3 days in the LMC (Acta Astr. 49, 341). An overview of contact binaries in galactic clusters and in the field, partly based on OGLE data, as well as an estimate of their space density, is given in AJ 116, 2998. 
A ROSAT survey of 8 systems indicated that two-temperature models for optically thin plasma describe well the observations (MNRAS 280, 627). EUVE and ORFEUS observations of nearby systems (AJ 115, 303; ApJ 502, 918; AAS 192, 5603) have shed even more light on the coronal emission in these systems. Future missions will certainly play a crucial role for our understanding of the outer structure of these systems, and may help to settle theoretical ambiguities about the equilibrium condition and the circulation pattern (see, e.g. A\&A 318, 171; A\&A 341, 567).

\section{MASSIVE CLOSE BINARIES}

\subsection{Massive Close Binaries: D.R. Gies}

The most massive binaries remain targets of considerable interest, and masses are now known for a significant number of eclipsing systems (ApJ 490,328; ASP Conf. Ser. 131, 401). The current record holder for most massive star in a binary is the Wolf-Rayet star in the $80 \mathrm{~d}$ binary, WR 22, which has a mass of $55 M_{\odot}$ (A\&A 347,127). The archive of the IUE satellite has proved to be a treasure source for radial velocity curves of a large number of close binaries containing O-stars (see Obs. 119, 16 and references therein), and tomographic and other reconstruction techniques have led to investigations of their individual component spectra (ApJ 518, 450).

Many massive close binaries are also members of triple or quadruple systems (AJ 115,821 ), and such systems are maybe the source of high speed runaway stars (MNRAS 255,423 ). The theory of the evolution of massive close binaries is compared to observational results in a new monograph The Brightest Binaries (Kluwer).

Radiatively driven winds are found in all massive, luminous stars, and in close binary systems the individual winds will collide in a bow shock which will wrap around the star with the weaker wind. The geometrical and physical characteristics of the collision boundary depend on the individual wind density and velocity laws, plus the system separation and orbital velocities, so there will be a great diversity in colliding wind properties among observed binaries. In some wide binaries the wind collision region creates non-thermal radio emission which is spatially resolved between the stars (MNRAS 289,10; AJ 115, 2047). The high temperature plasma formed in the shockfronts of colliding winds can lead to orbital phase variable X-ray emission (MNRAS 283,589), and this may explain the X-ray variations of the 5.5 year, very massive binary, $\eta$ Car. The shock boundary introduces both a density enhancement along its surface and a truncation of the otherwise spherical outflow, and this geometry creates orbital phase variations in the $U V$ wind lines and optical/IR emission lines. The high densities formed at the collision front during periastron in systems containing WC-type stars is the probable reason for their cyclic episodes of dust formation (ApJ 485,826). A binary interaction may account for the dusty pinwheel nebula observed around the Wolf-Rayet star, WR 104 (Nature 398,487).

Models of intermediate mass close binary evolution predict that mass gainers will be spun up to speeds close to the critical rotational velocity (appearing as Be stars) while the mass donors will appear as stripped down He stars, white dwarfs, or neutron stars. We now have the first example of a Be + He star system in the binary, $\phi$ Per (ApJ 493,440), and taken together with the well known Be X-ray binaries, there is clear evidence that some fraction of Be stars are created in binaries; whether all such rapid rotators are so formed remains unknown.

We can expect major advances in the study of close binaries in the next few years with the application of new ground and space-based optical interferometers which will angularly resolve most nearby systems. The combination of an astrometric orbit with a radial velocity curve will provide a full three-dimensional orbit, and if either a parallax or secondary velocity curve are available, then the individual masses and distance can be found. This will yield a large sample of stellar masses provided accurate radial velocity curves are available. Narrow-band interferometry at $\mathrm{H} \alpha$ and other emission lines should provide first maps of the circumstellar gas distribution for many binaries. 


\subsection{Advances in Massive Close Binaries and Future Expectations: V. Niemela}

Advances over the last three year:

- Optical CCD light curves of eclipsing OB binaries in the Magellanic Clouds are beginning to appear, which combined with proper radial velocity orbits will serve as first steps towards an empirical Mass-Luminosity relation in galaxies other than our own.

- Eta Carinae, the famous Luminous Blue Variable in our Galaxy, seems to be a close binary.

- The highest mass of a binary component, over 50 solar masses, seems to belong to a star with WN type spectrum in Carina OB1, HD 92740 (and note that stars with similar WN spectra are the most luminous members of the central cluster of 30 Doradus in the Large Magellanic Cloud).

Major unsolved problems are:

- The empirical Mass-Luminosity relation is only known in our Galaxy up to about 25 solar masses, and extrapolated to over 120 solar masses for other galaxies.

- We lack determinations of empirical masses of early $O$ type stars in binaries, which bears on our knowledge of the upper limit of stellar mass.

Impact of New Instrumentation

- Hopefully we will be able to study massive close binaries in their birthplaces with IR spectroscopy and photometry.

\subsection{Improved Physical Properties of Interacting OB CBS: H. Drechsel}

Drechsel and co-workers continue their efforts to derive accurate absolute dimensions for selected eclipsing binary systems. Spectroscopic and photometric observations of interacting OB-type systems are being collected and the MORO (MOdified ROche model) light curve analysis code (A\&A 294, 723), which can account for radiation pressure effects, is being used to analyze the observations. Recent solutions for two systems indicate that SZ Cam is a complex multiple system in which the third body is a close binary itself and with another visual companion showing evidence of duplicity as well (A\&A 332, 909). The other star, V606 Cen, belongs to the small group of early-type contact systems, and was found to be at nearly exact inner contact (A\&A 345, 531).

\section{THEORY OF CLOSE BINARY STARS}

\subsection{Late Stages of Close Binary Star Evolution: R. Taam}

In the past few years significant developments have taken place which have progressed our understanding of the late stages of close binary evolution. Included among them is the possible detection of a massive white dwarf in the close eccentric binary radio pulsar system PSR B2303+46 by van Kerkwijk \& Kulkarni (ApJ 516, L25). Originally considered a candidate for a double neutron star system, this observation provides evidence that the high eccentricity in this system $(\mathrm{e}=0.66)$ is insufficient to indicate that the system contains two neutron stars. In this case, the initial mass ratio of the system was probably close to unity so that first mass transfer stage was nearly conservative in order that the initially less massive star would be increased sufficiently to evolve to a neutron star. The second phase of mass transfer led to a common envelope, eventually leading to the formation of a white dwarf-neutron star close binary. Hence, the neutron star is formed after the white dwarf (necessary for imparting the high eccentricity) in this system. 
Mass and angular momentum loss are known to be important in the evolution of close binary systems. Recent work by King \& Ritter based on early massive Case B evolution (MNRAS in press) and Podsiadlowski \& Rappaport (astro-ph/9906045) based on Case AB evolution for the low mass X-ray binary system, Cyg X-2 suggests that significant mass loss takes place during the thermally unstable mass transfer in the system. Only by allowing for this mass loss can the present location of the observed companion and the mass accretion rate onto the neutron star be obtained consistently. The survival of the binary system to highly super-Eddington mass transfer rates suggests that the system can avoid the common envelope phase. The recent work by King \& Begelman (ApJ 519, L169) suggests that the common envelope phase can be avoided provided that the mass losing star is radiative and that the trapping radius of the accretion flow lies within the Roche lobe of the neutron star. It is envisioned that the matter is blown out of the system in a radiatively driven wind in a manner similar to that described by Blandford \& Begelman (MNRAS 303, L1).

There has been a significant increase in the detection of double white dwarf systems. Work by Marsh and collaborators (see astro-ph/9906100) has led to the detection of 15 such systems which primarily include helium white dwarf pairs. The increase in sample size and the determination of the white dwarf masses will provide significant constraints on the formation scenarios of these systems which will impact on population synthesis investigations.

In the future, the observations of binary $x$-ray sources will significantly enhance our understanding of the late stages of binary evolution. The study of transient sources in the Galaxy in both X-ray (RXTE, XMM, Chandra) and optical, promises to provide fundamental information on the mass and spin of black holes. In addition, the studies of binary sources in nearby galaxies will enable researchers to obtain a more complete census and determination of the space densities of many subclasses of systems for applications to population synthesis. Indeed, the study of the high energy transient systems will not only provide for the understanding of the systems themselves, but will also provide important insights on stellar evolution in close binary systems.

\section{CLOSE BINARIES IN GLOBULAR CLUSTERS: Luciana Bianchi}

Study of cataclysic variables (CVs) in globular clusters is important to constrain the twobody tidal capture theory. The formation mechanisms of CVs in dense cluster environments differ from those in the Galactic field, and are believed to involve interactions and tidal capture. Observations of the distribution of CVs within a globular cluster (GC) (core, where they would form by tidal capture, and outer regions, where relaxation time might be sufficient for a fraction of binaries close to $1 \mathrm{M}_{\odot}$ to survive to form CVs) will indicate the relative efficiency of the various mechanisms of CV production. New studies revealed differences between CVs in field and cluster environments. For example, there is preliminary evidence that CVs in clusters may have very low rates of mass transfer (see ApJ 516,250). Theory and recent observations also suggest that a higher percentage of cluster CVs are magnetic in nature (e.g., ApJ 350,L9; ApJ 516,250).

Binary systems are also important for their relevant role in the dynamical evolution of GCs. Two-body tidal capture theory predicts that all binary systems should be relatively common in Gcs, significantly greater in relative number than in the Galactic field. Among the most common binaries should be CVs, because of the high (presumed) abundance of white dwarfs in GC cores. Unfortunately, theory verification is still hampered by the lack of an unambiguous answer to the first simplest question: how many CVs are there in clusters? Searching for CVs in GCs is difficult (both to find candidates, and to confirm their nature). The high spatial resolution available with HST instruments have recently resulted in the detection of CV candidates in a handful of clusters, several of which have been confirmed by follow-up spectroscopy. Four CVs reported in the nearby, compact cluster NGC 6397 (ApJ 439,695; ApJ 516,250) and two CV candidates discovered in NGC 6752 (ApJ 473,L31) appear to be the (long sought) optical counterparts of low luminosity $\mathrm{X}$-ray sources. The 
outstanding mismatch remains between the large number of predicted CVs in clusters, and the scarce observational detections.

It is desirable for the future to progress in two ways:

1) observationally, to produce better statistics on CVs in clusters and to clarify the nature and measure physical parameters of faint X-ray sources. High spatial resolution of new technology telescopes and HST makes such progress possible.

2) theoretically, to further assess the stability of the products of tidal captures by WDs.

\subsection{Advances in Research in Symbiotic Stars: S. Kenyon}

Symbiotic stars are interacting binary stars where an evolved red giant and a hot companion are surrounded by an ionized nebula. Ultraviolet observations with IUE established this model as standard in the 1980's. Recent observations have concentrated on understanding the physical processes that produce symbiotic activity. In a modern triumph of atomic physics, Hans Schmid \& colleagues identified broad emission bands at $6825 \AA$ and $7082 \AA$ with O VI $\lambda \lambda 1032,1038$ emission, Raman scattered off neutral hydrogen in the outflowing red giant wind. After confirming this hypothesis with extreme ultraviolet and optical spectroscopy, they showed that the Raman scattered lines yield information on the orbital geometry of the binary and the structure of the wind in several of the brightest symbiotic systems. More general application of these techniques in the next decade will yield a better understanding of accretion and mass loss in the symbiotic class as a whole. Ken Hinkle and colleagues have shown that near-IR spectroscopy will become an important tool for deriving reliable orbits from radial velocities and for studying absorption line profiles of the red giants. Nearly all symbiotics are bright enough to measure high quality radial velocities with a near-IR spectrograph on a modest telescope. In addition to these 'general' advances, many groups made successful studies of individual or small samples of symbiotics using multi-wavelength data covering almost the entire electromagnetic spectrum. The new generation of X-ray (Chandra, XMM) and radio (SMA, MMA) telescopes will provide access to larger samples of symbiotics; future studies should yield common properties at these wavelengths that can be linked to the standard model developed to explain ultraviolet, optical, and infrared data.

On the theoretical side, Nussbaumer \& colleagues, Proga \& colleagues, and several other groups have developed detailed radiative transfer codes to model the symbiotic phenomenon. These groups all emphasize that accurate treatment of the continuum and line transfer is necessary to understand the energetics of accretion, winds, and any interaction regions. A major conclusion from these groups is that the hot component can ionize the inner portions of the red giant wind; this region emits most of the $\mathrm{H} \mathrm{I}$ and $\mathrm{He} \mathrm{I}$ line emission from the typical S-type symbiotic star with an orbital period of 2-3 yr. This idea was first proposed in the 1960s to explain observations of the reflection effect in AG Peg and several other symbiotics. The new models allow a better understanding of the red giant mass loss that powers a symbiotic binary. Rapid improvements in computers and the development of accurate Monte Carlo computer codes should enable routine 2-D and 3-D models for symbiotic stars in the next decade. These models, coupled with better ultraviolet data from $H S T$ and other space-based missions, should allow more detailed probes of the physics of symbiotic stars and a better understanding of mass transfer in evolved binary systems.

\section{CLOSE BINARIES WITH DEGENERATE COMPONENTS}

\subsection{Progress on Understanding CVs and LMXBs: J. Wood}

Observational and theoretical work on interacting binaries has produced many interesting results over the last couple of years. The first double degenerate polar was discovered, with an orbital period of 8 minutes - the shortest period known for any binary system. This is the first example of a He dominated radial accretion flow onto a white dwarf. 
Doppler tomography of accretion discs in dwarf novae during outburst have shown the presence of spiral patterns in the discs. These have been modelled theoretically as tidal density waves.

Rapid (a few seconds) spectroscopy has been done for a few CVs, for example, to investigate the spectra of dwarf nova oscillations. This pushes the limits of what is achievable with 4-m telescopes. The advent of Gemini will allow observations of fainter objects to be made, opening up the technique to many interacting binaries.

Phase-resolved, echelle spectroscopy of the evolutionary precursors to CVs (Post Common Envelope Binaries) has been obtained, allowing their secondary stars to be studied in detail. This is currently impossible for CVs and LMXBs where the accretion flow dominates the light. Again Gemini will allow this to be done.

The HST FGS has allowed the parallaxes of several CVs to be determined for the first time. These are some of the most distant objects ever to have a parallax measurement made. The measurements allow different methods of distance determinations for CVs to be checked. The DIVA satellite (Double Interferometer for Visual Astrometry) is planned which will allow the parallaxes of many CVs to be determined, removing a major source of uncertainty in modeling.

New X-ray observatories such as Chandra (imaging), Astro-E (spectroscopy) and XMM (spectroscopy/photometry) will significantly enhance the information that can be obtained for interacting binaries. XMM will also allow the first truly simultaneous X-ray and $U V$ observations, which are vital for understanding many aspects of CVs, e.g., boundary layers and the "Fe-curtain".

Theoretical work has long predicted that significant numbers of CVs should exists in globular clusters. After years of searching they are starting to be found with the HST. Four have been found in the globular cluster NGC6397, two in NGC6752, three in 47 Tuc and two candidates in Omega Cen. Theoretical calculations have shown that an instability caused by the irradiation of the disc by the central source can cause the disc to tilt and precess in the way that is observed in X-ray binaries, e.g., Her X-1. In CVs a coronal siphon flow at the center of discs has been calculated. This can explain the anomalous X-ray fluxes observed in some dwarf novae.

Theoretical calculations of advective flows in black hole LMXBs predict $\mathrm{Li}$ production via spallation and hence an anomalously high $\mathrm{Li}$ abundance in the secondary star. This is seen in soft X-ray transients and thus provides the best evidence so far as to the existence of advection dominated accretion flows.

\subsection{Cataclysmic Variables and Low Luminosity CBS: S. B. Howell}

The field of interacting binaries provides a heavenly laboratory for us to observe and study. Physical environments such as high gravity, temperature, mass, and magnetic fields are all presented to us in systems such as cataclysmic variables, low-mass X-ray binaries, and Algol systems. Detailed study of these extreme physical conditions are unavailable anywhere else within the $\mathrm{U}$ niverse.

Other authors in this report address the current state of knowledge in interacting binaries at high energies, especially exciting today with the fleet of recent and planned high-energy satellites in orbit. I will concentrate on two main aspects which I believe have made recent advances and which will draw attention in the future: observational study of low-mass, intrinsically faint, interacting binaries, and theoretical work on the evolutionary state of these binaries, particularly that of the mass losing star.

Presently, severe observational biases exist in our knowledge of the number and type of interacting binaries. The intrinsically bright systems are easily found, yet represent a very small fraction of the true population. In the field of cataclysmic variables for example, it is predicted by theory that about $90 \%$ of the systems in existence are still yet to be observed. Observational surveys, such as the Faint Sky Variability Survey, are working hard to remove this bias by obtaining variability and color information over a large area of the sky down 
to faint limits, near $\mathrm{V}=+25$. The results of such surveys will begin to appear within the next few years and will provide severe tests on the present day theoretical models.

The interest in these faint interacting binaries is not limited to the simple result of whether or not they exist, but also to how they form and physically behave. Interacting binaries form the only astrophysical laboratories in which accretion disks, high gravitational fields, high temperatures, mass loss, and stellar end-products can be studied both in detail and in concert. Most of the present-day astrophysical knowledge we have on such physical parameters comes from the study of some type of binary star system. The intrinsically faint systems allow us to discover the details of low mass transfer rates, optically thin accretion disks (for which there are currently no accurate models), and views of probably some of the oldest stars in our galaxy.

Theory says that many of the faintest systems are also the oldest, near 10 Giga-years or the age of the Milky Way itself. The degenerate stellar end-products (white dwarfs, neutron stars, and black holes) which make up the gravitational power plants, formed from massive stars early in the Galaxy's lifetime. The low-mass donor stars started their lives as normal main sequence stars and have been losing mass for the lifetime of the interacting binary. They now exist as some sort of unique type of low-mass red star having lost upwards of $90 \%$ of their initial mass. They have radii not much bigger then the planet Jupiter and surface temperatures of less than $2000 \mathrm{~K}$. There even may be interacting binaries which start their life with brown dwarf secondary stars.

It has been believed, that the mass donor stars in interacting systems should behave as single stars of the same spectral type. However, current work in this area shows that spectral type may not mean much for these mass-losing stars, and their rapid rotation rate, just over 1 hour for the shortest period systems, probably drives stellar activity, such as star-spots and active chromospheres, at a high level, far above that of even the most active single stars. Recent observational work using infrared spectra provides evidence for this, but much more work needs to be performed in this area. Once again we find that interacting binaries may provide our best astrophysical laboratory in which to study stellar activity cycles which may be driven to the extreme in the orbital period binaries.

\subsection{Some Ideas on Dwarf Novae and X-Ray Transients: C. Wheeler}

The last few years have seen many advances in the study of black holes in binary systems. The production of jets has cemented the similarity with quasars. On the theoretical side, the application of Advection Dominated Flows to these systems has been added to previous work with only thin, Keplerian disks and more recently with the question of whether strong winds blow most of the matter from the disk before it can accrete. Observations of transient outbursts have been shown to be more complex than can be understood with basic disk instability models that work for dwarf novae. Superhumps have proven to be ubiquitous in both ordinary close binaries and black hole systems and provide constraints on the masses and dynamics of the systems.

\section{NEW MISSIONS}

\subsection{The Impact of the GALEX Mission on Close Binary Stars: L. Bianchi}

In less than two years, the first $U V$ sky survey mission will begin. How will a $U V$ survey change our knowledge of binary stars?

GALEX (the GALaxy Evolution EXplorer) is a NASA SMEX (Small Explorer) planned to fly in 2001 for a 28 months mission, to perform imaging and spectroscopic surveys of the sky in the ultraviolet (see ESA SP-413,797) GALEX will perform the first all-sky survey in two $U V$ bands, down to $\operatorname{mag}_{A B}(2000 \AA)=21$, and deep imaging down to $\operatorname{mag}_{A B} 25-26$ of fields covering a total of 160 square degrees. GALEX will also perform slitless grism spectroscopy of the deep fields, covering the $1300-3000 \AA$ range with $10-20 \AA$ resolution and limiting magnitudes (at $2000 \AA$ ) from 20 to 23 . The angular resolution (about $4^{\prime \prime}$ ) will allow 
correct identification with optical counterparts of well over $90 \%$ of the $U V$ sources, giving astrometric precision of about $1^{\prime \prime}$ and classification of the $U V$ sources by astrophysical class without follow-up (Mem. SAI70,365; ASP Conf. Ser. 164,194).

How will the $U V$ all sky survey change our knowledge of close binary stars? Our current understanding of late stages of stellar evolution, such as WD, and close binary evolution, is limited by the paucity of objects. Systems with accretion disks often radiate a large fraction of their luminosity in the $U V ; U V$-to-optical photometry will allow an unbiased census of these objects and reveal their nature. $U V$ fluxes, combined with optical fluxes, will be a sensitive diagnostic to reveal and measure the presence and mass exchange in accretion disks, and the hot (optically faint) companions. Flux limited, unbiased samples of CVs and LMXRB will be representative of the evolution of the accretion disk, and of the hot components of these evolved binary systems. Also, the GALEX imaging survey will detect several thousand CVs and WDs (as well as nuclei of PN); two $U V$ bands, plus optical colors of counterparts in optical surveys will allow a statistical classification by object type, and unbiased statistically significant luminosity functions. For several hundreds of these objects GALEX will provide $U V$ spectra that will allow better modeling and refined temperature and extinction determination.

Post main sequence evolution, and common envelope phases for binary objects, are very fast evolutionary stages, thus a significant numerical increase (with parameters determination) for these classes of objects will constrain the detailed path for stellar evolution in these observationally-elusive phases, scenarios of neutron star formation by accretion, etc.

GALEX surveys will also contain serendipitous variability information, thanks to the photon counting detectors. Time scales will vary from very short (source travelling through the field of view during a scan) to days/months (repeated scans to achieve the limiting magnitude)depending on the actual survey observation strategy for the different sky locations. Time resolved magnitudes and spectra will be important to discover accreting binary systems, and eclipsing systems. Several hundreds should be detected by the surveys.

\section{ADVANCES \& THE FUTURE OF RESEARCH IN CBS}

\subsection{Prognosis for the Future of Close Binary Research: Y. Kondo}

The study of close binary stars consists primarily of: (a) understanding the physical properties of the components, (b) interactions between the two stars, and (c) evolutionary processes that would involve (a) and (b). For the first two centuries since the work of John Goodricke, and especially since the advent of photometry and spectroscopy, we have made significant progress in (a). For the past half century, pioneered by people like Otto Struve and Gerald Kuiper, we have also been making progress in (b). The 1960s and 1970s saw an introduction of speculative ideas on the evolutionary processes that were sometimes, or even often, based more on conjectures than on sound physical principles (e.g., totally conservative mass exchanges between the two components based on the so-called Roche lobe overflow idea); not withstanding the appeal of the 'models' based on simplistic restricted three-body approaches (that are inapplicable to real binaries), magnetohydrodynamic gas flows in close binaries are not easily susceptible to mathematical modeling. With the advent of satellite observatories, we have started gathering new spectroscopic and other data to study directly the gas flows in interacting binary systems; in stars thus far observed (e.g., U Cep), we have seen gas outflows out of the binary as well as accretions to the companions; we have also observed binaries where both components are engulfed in high-temperature gases (e.g., $\beta$ Lyr) that are leaving the binary systems, and interactions of winds from both stars in some binaries (e.g., $\iota$ Ori).

Interferometric telescopes, both on Earth and in space, promise to give us direct imaging of what is really happening in nearby interacting binary stars instead of relying on speculations. For example, the Moon is an excellent optical bench in vacuum, where diffraction-limited imaging is achievable. Using optical cables or other means, we can in principle connect an array of telescopes with a long base-line; with a base-line of $50 \mathrm{~km}$, 
we can achieve a resolution in the visible light of $10^{-10}$ radian. This will correspond to a resolution of $10^{10} \mathrm{~cm}$ at a distance of 100 light years (roughly $10^{20} \mathrm{~cm}$ ). The solar diameter is a little over $10^{11} \mathrm{~cm}$; this resolution then is equivalent to the tenth of the diameter of a typical star like the Sun, which is sufficient to start actually observing gas flows between the two stars.

The majority of stars in the sky appear to be members of double or multiple systems; hence, understanding the physical properties and evolutionary processes in binary stars is essential for comprehending the nature of the galaxies and the Universe as a whole. In addition, most astrophysically interesting objects appear to involve binary systems, e.g., cataclysmic variables (including Type Ia supernovae) and X-ray binaries involving white dwarfs, neutron stars and black holes. We stand on a door step to an era when all of the above-mentioned three areas of research become susceptible to quantitative scientific analysis.

\subsection{CBS Census from Microlensing Surveys: E. F. Guinan}

Close binaries are ubiquitous and contain examples every type of stellar astronomical object from pre-main-sequence stars, main sequence, giants, super giants, white dwarfs, neutron stars, black holes and more recently, even brown dwarfs and planets. Less than a decade ago about 4000 close binaries were known in the Galaxy with an additional 200 or so systems identified in the Magellanic Clouds and M31. However, now over ten thousand close binaries have been discovered; more half of them were detected over the last few years! The number of close binaries has increased dramatically, primarily from the serendipitous discovery of thousands of eclipsing binaries from microlensing surveys conducted by the EROS, OGLE, and MACHO searches. Nearly a thousand close binaries with apparent magnitudes as faint as +17 th mag have been detected in the direction of the Galactic bulge from OGLE (ACTA 48,1 ). This brings the number of CBS known in the Milky Way to about 5500 systems depending on how one counts them. At the same time there has been an amazing, nearly one hundred-fold increase in the number of CBS detected in the Magellanic Clouds. OGLE II (ACTA 48, 563) has recently detected nearly 1500 eclipsing binaries in the SMC in the central 2.4 square degree field of the galaxy, while at last count, over 3500 eclipsing binaries have been identified in the LMC as a by-product of the MACHO surveys (Kem Cooke, priv. comm.). It is estimated that the MACHO program alone will detect over 10,000 LMC and SMC eclipsing systems over the next few years. Moreover, eclipsing binaries are being discovered in M31 and M33 by the DIRECT project (AJ 115, 1016; AJ 115, 1894) and in M31 by a Spanish group (Ribas, Priv. Comm.). These newly discovered close binaries have only recently started to be mined for the valuable information they contain. For example, as pointed out by some of the contributors, detached double-line eclipsing binaries are now being utilized as accurate "standard candles" to gauge the distance to the LMC (ApJ 509, L21, L25). As more of these are done, it may be possible to pin the down the distance to the LMC to an accuracy of better than $2 \%$. Also, if this method proves reliable, it may be possible to probe the structure and dynamics of the the LMC and SMC with eclipsing systems.

\subsection{Recent Developments in CBS Research: J. V. Clausen}

- The interesting work that Dan Popper was doing for several years after his retirement on G-and K-type binaries is noteworthy. With his latest publications it is clear, that the existing binary dimensions pose a dilemma for current stellar models. Reason yet unknown! I would like to mention that Dan Popper died on September 9, 1999. His contributions to close binary studies over his long career are significant. He will be missed by his friends, the astronomy community, and, in particular, the Close Binary Star community.

- There appears good evidence from recent studies of specially selected eclipsing binaries that the convective-core overshooting parameter (or whatever describes what is going 
on in the star's core) is not constant with the stellar mass (ASP Conf.Ser.173,41). Also in the PhD thesis of Ignasi Ribas (Un. of Barcelona, 1999), eclipsing binary dimensions plus stellar models have been used to obtain a fairly well defined (compared to other methods) relation between the helium abundance $(Y)$ and metal abundance $(Z)$, or $\delta(Y) / \delta(Z)$.

- Another recent development related to eclipsing binaries is the determination of an accurate distance to the LMC using an eclipsing binary star (LMC-HV2274) as a "standard candle" (ApJ 509, L21) This is important by itself, but also for future progress. One might hope that the astronomical community now becomes convinced that this method actually works, and is needed to calibrate, e.g., Cepheid P-L relations to obtain distances to distant galaxies without possible serious systematic errors (and thereby leading to an error in the value of $\mathrm{H}_{0}$, for example).

- I am also impressed with the OGLE work on SMC (and LMC), but as far as I know, the eclipsing binaries are not yet published. After all - the OGLE SMC eclipsing binaries are an important supplement to the EROS and MACHO material for LMC. Binary studies in both MC's are needed, e.g., to check metalicity dependence of Cepheid P-L relations which are also important for determining the extent and structure of SMC.

- Also noteworthy is the increasingly accurate masses being determined for(mostly) Ftype binaries through the "TODCOR" work at CfA. Also, the CfA group is obtaining interesting results on visual binaries that may be of interest to the CBS community.

\subsection{The Future of Research in Close Binaries: J. Sahade}

The President of Commission 42 has asked the Commission's O.C. members to put forward their feelings regarding the "progress that has been made in the last few years and address the advances that might be expected during the next decade or so with new instrumentation and telescopes (both on the ground and in space), more powerful, faster computers, and with improved physical models, etc." Since this is so, I would like to take the opportunity to bring up once again the need to think in building modern telescopes with special purposes in mind. One such special purpose is to advance our knowledge of interacting binaries and for this we need to have telescopes that would devote continuous periods of time to the observation of particular objects (or to tackle specific problems) both photometrically and spectroscopically in the spectral regions. This can be done with present day technology. The large telescopes now being operating or planned will be mainly devoted to other types of problems and will never assign appropriate amount of observing time to tackle the problems that are the concern of Commission 42. Therefore, I would suggest that, under the encouragement of Commission 42, possible partners should be found to finance the erection and operation, at appropriate places, of adaptive optics telescopes to be engaged in the observation of appropriate interacting binaries, in the photographic and IR regions of the spectrum. A special group within the Commission could undertake the task.

I have brought up this issue as a speaker at a couple of general astronomical meetings, without having any reaction on the part of the audience, but they were not specialized meetings on the specific subject of interacting binaries, There exist, in many countries, groups of astronomers that are particularly interested in the field of Commission 42 and should be potential champions of such an enterprise. Perhaps the IAU, together with UNESCO and perhaps ICSU, could sponsor it, start investigating the possibilities and lay the first stone.

\subsection{Gazing into the Future of Close Binary Stars: E. Olson}

Maybe we retired chaps are supposed to sit around and pontificate about the futures of our specialties. I haven't and won't, but here are a few ideas that come to mind. 
I have a fantasy. Extragalactic distance indicators finally fail to break the $10 \%$ barrier in Hubble Constant accuracy. In their final blaze of glory, detached eclipsing binaries in external galaxies (something I think you know a lot about) are the final hope. By the year 2004 the Keck telescope is busy measuring radial velocity curves of double-line binaries in such galaxies. After a year or two, observational cosmologists are able to announce a Hubble Constant accurate to... get this... 5\%! They declare victory and withdraw. The field of binary stars begins a precipitous decline...

Slightly more seriously, we seem to know some things about binary star formation, but puzzles remain. Common envelope evolution seems to be important to the formation of cataclysmics and X-ray binaries. RS CVn binaries will likely help in understanding solar activity. Interest in binaries will continue be proportional to at least the fourth power of accretional energy density. We will have to become adept at $3 \mathrm{D}$ radiation and magnetohydrodynamics, I guess.

In my own remote binary outback, I've always hoped that long-period Algols, which often have genuine (but low-density) accretion disks, might shed some light on other AD situations. I wish more strongly this might be so, after reading Ed Sion's masterful account of white dwarfs in cataclysmic binaries (PASP 111,532). It hasn't happened yet. Sure, Algol disks are almost invisible in the continuum, but $\mathrm{H}-\alpha$ disk emission and $\mathrm{O}$ I 7774 absorption are very strong and, with proper modeling, would be incisive diagnostics of disk structure. Progressive eclipses of, and by, disks potentially add a lot of detail. Gainer mass and angular momentum accretion from the inner disk to some extent parallels that in non-magnetic cataclysmics. There is some chance of detecting the expected differential gainer rotation during primary eclipses (we have some observations but probably not yet at sufficient spectroscopic resolution). And I'm fairly convinced of systematic differences between spectroscopic and photometric rotations that may suggest angular momentum assimilation by gainers.

I hope to live long enough to exploit our data.

I come back to the comment a famous binary star expert made in the context of space mission: "How about a mission to determine limb darkening?"

Edward F. Guinan

President of the Commission 